\title{
New Horizons in Geriatric Medicine Education and Training: The Need for Pan-European Education and Training Standards
}

\section{Introduction}

The global population is rapidly ageing and, whilst this should be celebrated as evidence of the efficacy of modern medicine, one consequence is an increase in those living with multi-morbidity and frailty[1]. The resultant impact on health and social care systems is well recognised. The recent World Health Organisation (WHO) Report on Ageing and Health called for changes to health policies for ageing populations[2], specifically for health systems to align themselves to the older population that they now serve and for long-term care systems to be developed. There is compelling evidence from large systematic reviews[3, 4], that Comprehensive Geriatric Assessment (CGA) is the most effective way to provide healthcare services for this population. CGA has been shown to increase patients' likelihood of being alive and in their own homes after an emergency admission to hospital.

CGA can be defined as a "multidimensional interdisciplinary diagnostic process focused on determining a frail older person's medical, psychological and functional capability in order to develop a coordinated and integrated plan for treatment and long term follow up"[5]. Geriatricians are medical specialists with expertise in the healthcare of older people, and have a key role in delivery of CGA - central to this is geriatricians' ability to manage multi-morbidity[6]. The essence of managing multi-morbidity is having the awareness that older people require a different type of care to their younger, more physiologically robust counterparts. Clinical and biological signs of disease are different in older people with frailty. Diseases are often revealed by non-specific presentations, or via atypical presentations due to abnormal physiological responses to acute illness - a thorough clinical assessment is therefore mandated. CGA is not just a detailed clinical assessment performed by a geriatrician - it moves beyond identification of the patient's needs to the delivery of a multifaceted intervention that seeks to restore wellbeing, participation in activities and independence, and to ameliorate disability and distress. Despite the existence of highquality evidence surrounding CGA, there remains disparity between what we should be doing and what we are doing. The reasons for this 'know-do' gap are likely to be manifold but a failure to generate and spread expertise has been recognised as a likely barrier to implementation[7].

This paper aims to make a case for the development of pan-European education and training standards for the field of geriatric medicine. Whilst we acknowledge that ageing presents healthcare systems with challenges on a global scale, and that geriatric medicine may offer potential solutions[8], this paper will 
consider Europe specifically. The free movement of doctors within the European Union and the collaboration of medical specialties to develop common training standards through the Union of European Medical Specialties (UEMS) provide both a need and an opportunity to better standardise training pathways in geriatric medicine across Europe. Against this background this paper aims to make a case for the development of pan-European education and training standards in geriatric medicine. This should not be seen as discrete from, or in competition with, attempts to develop better international consensus in this arena.

In this paper, we start by describing the substantial challenges which face the implementation and delivery of geriatric medicine in a systematic way across Europe by outlining the differences in how geriatric medicine and general internal medicine are structured and delivered. We then go on to describe the opportunities for geriatric medicine, and how a better understanding of core competencies in geriatric medicine amongst non-geriatricians, has potential to bridge existing gaps in service provision across the continent. Finally, we consider how work can proceed to teach sufficient numbers of doctors and health professionals in the core knowledge, skills and attitudes required to do this. 


\section{Post-graduate Geriatric Medicine training across Europe}

The number and density of geriatricians differs widely across Europe[9] and in some countries geriatricians simply do not exist[10]. Recent surveys have demonstrated wide variability in postgraduate geriatric medicine training[11-13]. Firstly, in 5 of the 31 countries surveyed, geriatric medicine was not recognised as a specialty or a sub-specialty[11]. Two thirds of European countries did recognise geriatric medicine as an independent post-graduate specialty, with the remainder viewing it as a sub-specialty, mainly of internal medicine[11]. Nearly all the countries offering postgraduate training in geriatric medicine have written, competency-based curricula covering different learning domains[11]. For those countries that do recognise geriatric medicine as a distinct specialty, there is considerable variability in the length of postgraduate training[12]. Currently, the minimum length of training required by the European Directive on medical speciality training is 4 years post-qualification from medical school[12]; the length of geriatric medicine specialty training programmes across Europe ranges between 4-9 years. We contend that 4 years of postgraduate training is insufficient to become a specialist in geriatric medicine. It is important to highlight the limited time dedicated to geriatric medicine topics in undergraduate medical education and also its variability across Europe[10, 14]; these findings add weight to the argument for the need for consensus on post-graduate training length, since the baseline competencies of new trainees is likely to be variable. There is also notable variety in the regulatory bodies that are responsible for post-graduate geriatric medicine training across European countries - these include national medical associations, health ministries and universities. Barriers to recruitment to a career in geriatric medicine have also been identified. Trainees, although identifying with the intellectual challenges and the "mission" of geriatric medicine, cite financial and status-related issues as deterrents to a career in the specialty[15, 16]. In some countries, a perceived lack of research opportunities in ageing and clinical research has also been identified as a deterrent to a career in the specialty.

Some reviews of medical training programmes have highlighted the central role of general (internal) medicine $(\mathrm{G}(\mathrm{I}) \mathrm{M})$ training, with focus on the recognition and management of older people living with frailty and functional dependency[17]. A further barrier is the progressive sub-specialisation of singleorgan/system specialists with the argument that the needs of patients with multiple pathologies would be best served by ensuring they have access to a variety of super-sub-specialists[17], and that to maintain their sub-specialist skill-set, ongoing participation in the acute, unselected medical take is not viable, since the demands of continued involvement would interfere with delivery of their sub-specialism. The upshot of these arguments is the assertion that generalism is the preserve of hospitalists, acute physicians, $\mathrm{G}(\mathrm{I}) \mathrm{M}$ 
physicians, geriatricians and general practitioners[18]. Commitment to the general medical on-call rota and the associated burden of service provision is, however, perceived by some trainees as leading to reduced academic and training opportunities - this was considered an important downside to a career in the specialty[16]. In some countries the progressive sub-specialisation of single organ specialists has resulted in the formation of a service gap, which has subsequently been filled by newly conceived specialties such as Acute Medicine[19]. At best, these specialties have recognised the knowledge and skillsets required to care for older patients, and are up-skilling appropriately. At worst, there is failure to acknowledge and integrate the evidence-based method of providing care for older people with frailty (CGA) into their clinical practice, and failure to work collaboratively across boundaries between specialties. Given the scale of the challenge that ageing presents, a pragmatic approach is required clinicians looking after older people must be fully trained to do so, enabling non-geriatrician to develop competencies outlined in the curriculum that may enable the workforce to become more 'geriatricianly' and may result in better care of older people. 


\section{The scope of Geriatric Medicine practice across Europe}

In recent years, geriatric medicine has diversified and become increasingly sub-specialised[20] (Figure 1).

Figure 1: The Scope of Geriatric Medicine Practice across Europe

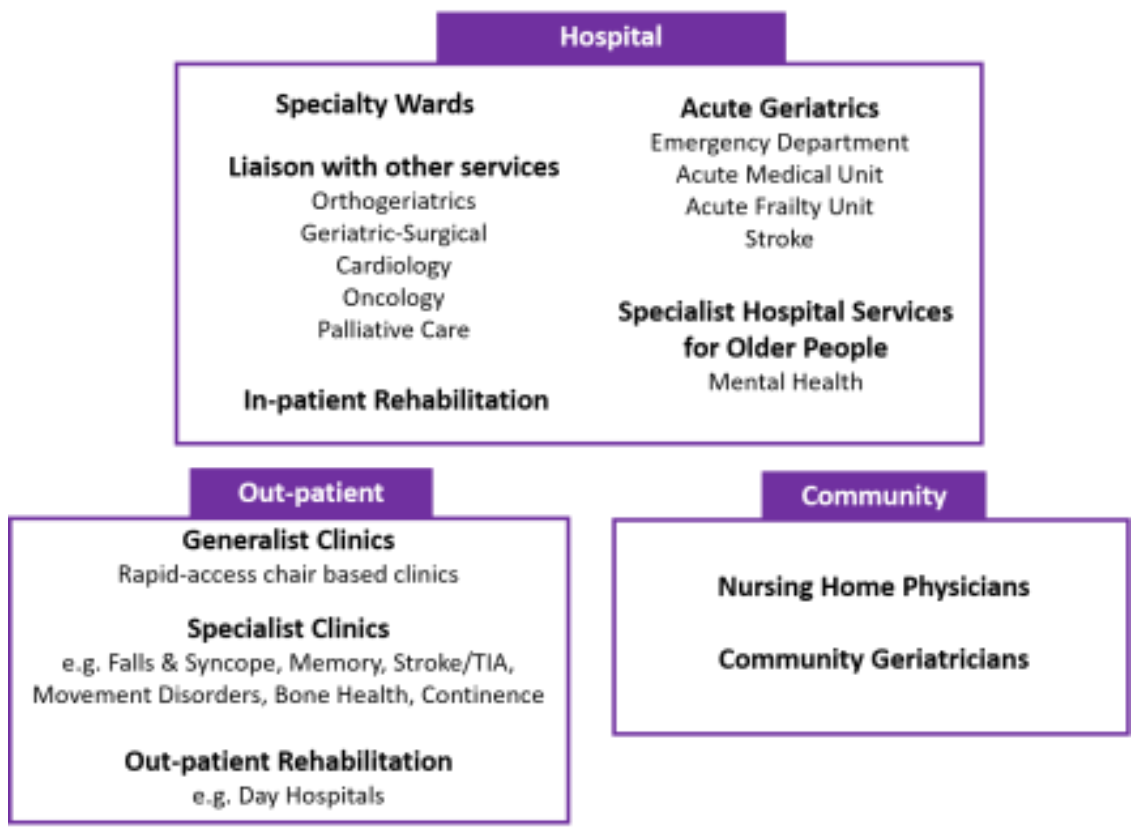

\section{Hospital}

Acute Geriatrics: There has been progressive integration of geriatricians into acute hospital environments such as emergency departments[21], Acute Medical Units[22] and Acute Frailty Units[23]. The extent to which this model ('interface geriatrics') has developed varies across Europe - in many countries (e.g. Germany, Norway, France) most geriatricians have partial roles within acute care, in some (e.g. Belgium) geriatricians are almost entirely within acute care[24]. Belgium's "Care Programme for the Geriatric Patient", introduced in 2007, is notable for mandating CGA for all patients over 75 years admitted to hospital with a 'geriatric profile', determined by an admission screening tool. Such initiatives allow earlier recognition of frailty, enabling timely initiation of CGA. Such approaches have shown reductions in mortality[25] and bed occupancy, without affecting re-admission rates or requiring additional resources[23].

Specialty Wards: The evidence base for CGA is strongest for specialty ward-based care[3, 4] where the majority of published studies have described geriatrician involvement. A Cochrane review of such 
interventions showed that patients who underwent CGA were more likely to be alive and in their own homes, less likely to die or experience deterioration and more likely to experience improved cognition[26]. Care in specialist environments may also incur lower total inpatient costs when compared to 'usual' care[27].

Specialist Hospital Services for Older People: Geriatricians have led on the development of specialised delirium units which have resulted in shorter duration of delirium and reduced length of stay[28]. Many geriatricians work alongside Mental Health Liaison services for older adults with some evidence of positive outcomes, although provision of such services remains highly variable[29].

Rehabilitation: Many of the concepts underpinning the rehabilitation process overlap with the principles of CGA, hence geriatricians across Europe often provide the medical expertise within a multidisciplinary rehabilitation team. In-patient rehabilitation, specifically designed for older patients, has been shown to improve outcomes related to function, discharge destination and mortality[30]. Delivery of out-patient rehabilitation is also seen and has been demonstrated to be efficacious[31, 32].

\section{Liaison with other services:}

A number of models of orthogeriatric care exist, but the joint care model (between geriatrician and orthopaedic surgeon on a dedicated orthogeriatric ward) has been shown to reduce inpatient mortality and decrease length of stay[33]. Recent work in Sweden[34] with a hip fracture patient cohort demonstrated significant reductions in mortality and readmission rates and provides compelling 'realworld' evidence for the efficacy of geriatric care at the healthcare system level.

In contrast to orthogeriatrics services, geriatrician collaboration with surgical teams is in its infancy, tending to be reactive, focussing on emergency cases, and lacking CGA as the guiding principle[35]. Metaanalysis of data to establish the efficacy of pre-operative CGA is not yet available, but in older patients undergoing elective surgery, preoperative CGA has a positive impact on postoperative outcomes, such as medical complications and length of stay [36].

Collaborative working between geriatricians and cardiologists is increasingly seen, such as coordinated care of heart failure patients and CGA provision pre-invasive interventions[37]. Links between oncology and geriatric services are developing at pace, and are becoming increasingly prevalent and relevant across Europe. Evidence exists demonstrating that variables considered within CGA can predict morbidity and 
mortality in older patients with cancer[38], but the potential mortality benefit of CGA has not yet been demonstrated. Many geriatricians have interest and skills in palliative care due to the frequency with which end of life care is encountered in a geriatric medicine setting.

\section{Out-patient}

Increasingly, European geriatricians offer rapid-access, 'chair-based' clinics for older people, equivalent to Ambulatory Care clinics typically provided by Acute Medical teams. This model can prevent older patients being referred to Emergency Departments and may lead to a reduction in emergency, unscheduled admissions.

Geriatricians are frequently involved in the delivery of specialist outpatient services, including falls clinics[39], memory clinics, transient ischaemic attack clinics, Parkinson's disease (PD) services[40], bone health clinics[41] and continence care[42]. Across Europe variation is seen in how such services are delivered. PD services, for example, are often neurology-led, yet the multi-system nature of the condition means an approach analogous to CGA may be preferable for older PD patients living with frailty. Stroke physicians can train via a variety of different routes, with geriatric medicine and neurology being the most common - again, there is variation across European countries in what constitutes the 'usual' training route.

\section{Community}

Geriatricians are increasingly working across the boundary of primary and secondary care; some work solely in the community, but the majority have commitments in both settings. In the UK, community geriatricians now constitute the largest group of traditionally hospital-based consultants working within the community[43]. There is appetite across Europe for greater emphasis on the recognition of frailty in the community setting and for the implementation of community-based interventions. The efficacy of CGA in community settings has been demonstrated in a large systematic review[44], although a review of CGA at the interface between community and acute care did not show clear benefit[45]. The challenge here is separating absence of evidence, from evidence of absence and considering what alternative model one would propose to health and social care sectors hungry for solutions, if not one which is informed by expertise, is multi-domain and multi-professional in approach, and is co-ordinated and iterative[7]. Nuanced understanding of the health needs and requirements of older people is essential to this debate and this is a profound argument in favour of expanding training in care of older people. 
Care home residents are recognised as being frail, dependent and vulnerable, with high levels of multimorbidity and complexity[46]. Long-term care services may be provided in a variety of settings and a wide range of national arrangements and national labels exist. Wide variation in European nursing home practice and long-term care is seen, which may in part be explained by a lack of training amongst care home staff and medical staff[47]. Regular input from geriatricians into nursing homes can reduce emergency admissions [48] and in the Netherlands care home medicine has developed into an established medical specialty[49], however barriers to implementation exist across Europe, including insufficient geriatricians and sometimes, resistance from other healthcare professionals to geriatrician involvement. 


\section{The need for pan-European Geriatric Medicine Education and Training Standards}

Despite the evidence base that underpins the specialty there is wide variance in European geriatric medicine practice[12]. We acknowledge that the process of translating the entire suite of geriatrician-led interventions into standard practice across Europe is challenging, and is likely to evolve differently in countries depending on historical workforce concerns, models of healthcare provision and prevailing attitudes amongst doctors, policymakers and society at large. In European countries where geriatric medicine is less well established, a lack of understanding of what geriatricians can add to patient care has contributed to insecurity of the specialty. The significant heterogeneity in geriatric medicine training programs across Europe is likely to further compound this insecurity. Efforts are underway to better understand the similarities and differences between training across Europe, through the recent development (using a modified Delphi procedure) of a geriatric postgraduate education assessment instrument[50]. We contend that the heterogeneity seen in both service provision and post-graduate training in geriatric medicine are inextricably linked, and argue that to safeguard the future of the specialty across Europe, there is a need for harmonisation of post-graduate training.

It is vital that clinicians responsible for the care for older people are competent and formal curriculum development is an essential in ensuring competencies and safeguarding high quality care. Development of a such a curriculum may also help to address the variance in training times across Europe. Moving towards outcome-based, rather than time dependent, curricula will mean that decisions about a trainee's competence will be based on what they can do, rather than how much time they have spent in post. This may, in time, enable a more nuanced understanding of what the typical training time ought to be. Curriculum development is critical to effective delivery of post-graduate education in the core knowledge, skills and attitudes required in geriatric medicine. In addition, achieving pan-European consensus on a geriatrician's competencies may help to counter the uncertainty within some societies about the specialty. To advance the evidence base the specialty does needs to train more medical researchers and to encourage more young geriatricians to pursue academic careers[51]. Embedding exposure to research in postgraduate training, through its inclusion in the curriculum, may help drive this process. Consensus on the competencies required within such a curriculum would also enable countries where geriatric medicine is less well established to benchmark their training programmes and to modify the content to a minimum agreed level. Examples of such initiatives do exist in other countries around the world. The American Geriatrics Society/Association of Directors of Geriatric Academic Programs produced a set of 'curricular milestones', to provide clarity about what a graduating geriatric fellow ought to be able to 
do[52]. In Europe, a collaboration between the European Union Geriatric Medicine Society (EUGMS) education special interest group (SIG), the European Union of Medical Specialists (EUMS)-Geriatric Medicine Section, the European Academy for Medicine of Ageing (EAMA) and the International Association of Gerontology and Geriatrics (IAGG) has started a consensus development process (modified Delphi), as a vehicle for developing the curriculum. However, there are a series of challenges which must be overcome. Firstly, the variation within European geriatric medicine practice needs to be acknowledged. International variation in health and social care structures is arguably inevitable, but we contend that it brings with it positives too. Undertaking a collaborative curriculum development process with European colleagues forces clinicians and educators to look critically at their own and others practice, and in doing so, unlocks the potential to seed gold-standard training and practice further afield. The feasibility of such a process has previously been demonstrated; recently, an international modified Delphi technique was used to achieve consensus on a European undergraduate curriculum in geriatric medicine[53]. This undergraduate curriculum has now been accepted in principle by all the EU countries and medical schools are being encouraged to map their curricula to this framework. Second, the wide variation in training time must be addressed. If trainees in one country are given less training time than their counterparts in other countries, but are still expected to achieve the same competencies, the curriculum would be devalued and less likely to be implemented. Thirdly, once developed the curriculum needs to be implemented widely; the multi-agency collaboration described above provides a vehicle for achieving this. In addition, the EUMS has previously established a standard procedure for the development and formal approval of European Training Requirements in each specialty field of medicine; such standards can therefore be readily applied to geriatrics.

The development of such a curriculum may also bring wider benefits. [2]. As well as increasing the number of geriatricians, there is a need to ensure that all healthcare professionals grasp the basic principles of care of older people, given the needs of the ageing population and the scale of the challenge facing health services[54]. A degree of non-specialist familiarity with CGA is needed to assist in the proper treatment of non-complex older people and the identification of those that need all the skills of an MDT led by a geriatrician. Geriatricians, as educators, have a central role in assisting other professional groups to develop the battery of skills required for effective care of older people. Geriatricians also have a role to play as health care advocates through challenging negative societal preconceptions about older people and by serving as healthy aging experts. Multi-modal preventive care strategies, or 'healthy ageing' programmes, with geriatrician input have shown great promise amongst healthy older individuals improved health behaviours and survival benefit have been demonstrated [55]. 
Whilst it will be used as a road-map for training a specialist geriatrician, a pan-European curriculum for post-graduate geriatric medicine training could also be used to identify selected core competencies required by other professional groups who regularly see frail older people. An example of how geriatric medicine expertise has inculcated post-graduate training in another field can be seen with the panEuropean development of a validated curriculum in geriatric emergency medicine[56], through collaboration between Geriatric and Emergency Medicine (led by EUGMS education SIG with and support from the EUMS).

Whilst we acknowledge that variability is likely to exist in other specialties across Europe we contend that this is not at the same level as encountered in geriatric medicine. This is likely due to the relative newness of geriatrics as a specialty, its general nature, which means that many non-geriatricians find it difficult to comprehend where it fits in, and the way in which it has been implemented in response to specific healthcare challenges in different countries. Thus geriatricians, more than most, need to make efforts to achieve consensus around core principles, including those of what needs teaching and when. 


\section{Conclusion}

The variance in geriatric medicine training and practice across Europe has been described; in doing so, it is evident that the specialty occupies different roles within different healthcare systems across Europe. In some countries geriatric medicine is deeply embedded into the workings of the healthcare system and is recognised as adding value to patient care; in others, there is a failure to recognise the potential value that geriatricians can add to patient care. Of concern, is that in some countries the discipline faces an existential struggle for its identity as a distinct specialty. Tackling this problem requires a multi-faceted approach, that includes engaging and educating senior policy makers, healthcare system coordinators and governments, about the added value that our specialty can bring. A major contributory factor to the insecurity of the specialty in some countries however, is the significant heterogeneity in geriatric medicine training programs across Europe. To counter these threats to the specialty this paper discusses the breadth, variety and, where available, the evidence base that underpins the specialty's efficacy. To safeguard the future of the specialty across Europe, we contend that there is a need to strive towards harmonisation of post-graduate geriatric medicine training through the establishment of pan-European education and training standards in the specialty. In future, establishing a consensus European curriculum may provide a useful starting point for global discussions about what a world-wide curriculum for geriatricians might consist of.

DECLARATION OF FUNDING: None

CONFLICTS OF INTEREST: None

ETHICAL APPROVAL: Not required 


\section{References}

1. Romero-Ortuno R, Fouweather T, Jagger C. Cross-national disparities in sex differences in life expectancy with and without frailty. Age and Ageing. 2014;43(2):222-8.

2. World Health Organisation. World report on ageing and health. 2015 [cited 2016 10th November]; Available from: http://www.who.int/ageing/publications/world-report-2015/en/.

3. Stuck AE, Siu AL, Wieland GD, Rubenstein LZ, Adams J. Comprehensive geriatric assessment: a meta-analysis of controlled trials. The Lancet. 1993;342(8878):1032-6.

4. Ellis G, Whitehead MA, Robinson D, O'Neill D, Langhorne P. Comprehensive geriatric assessment for older adults admitted to hospital: meta-analysis of randomised controlled trials. BMJ. 2011;343:d6553.

5. Rubenstein LZ, Stuck AE, Siu AL, Wieland D. Impacts of geriatric evaluation and management programs on defined outcomes: overview of the evidence. J Am Geriatr Soc. 1991;39.

6. Hafner K. As Population Ages, Where Are the Geriatricians? The New York Times. 2016 26th January.

7. Gladman JRF, Conroy SP, Ranhoff AH, Gordon AL. New horizons in the implementation and research of comprehensive geriatric assessment: knowing, doing and the 'know-do' gap. Age and Ageing. 2016 March 1, 2016;45(2):194-200.

8. Michel J-P, Cha HB. Filling the Geriatric Education Gap Around the World. Journal of the American Medical Directors Association. 2015;16(12):1010-3.

9. Kolb G, Andersen-Ranberg K, Cruz-Jentoft A, O'Neill D, Topinkova E, Michel JP. Geriatric care in Europe - the EUGMS Survey part I: Belgium, Czech Republic, Denmark, Germany, Ireland, Spain, Switzerland, United Kingdom. European Geriatric Medicine. 2011;2(5):290-5.

10. Michel J-P, Huber P, Cruz-Jentoft AJ, a representative of each surveyed c. Europe-Wide Survey of Teaching in Geriatric Medicine. Journal of the American Geriatrics Society. 2008;56(8):1536-42.

11. Singler K, Holm EA, Jackson T, Robertson G, Müller-Eggenberger E, Roller RE. European postgraduate training in geriatric medicine: data of a systematic international survey. Aging Clinical and Experimental Research. 2015;27(5):741-50.

12. Stuck $A E$, Jónsdóttir $A B$, Singler $K$, Roller RE, Holm $E A$, Masud $T$. The length of postgraduate training for geriatric medicine in European countries: an update for the year 2015. Aging Clinical and Experimental Research. 2016;28(1):169-70.

13. Reiter R, Diraoui S, Van Den Noortgate N, Cruz-Jentoft AJ. How to become a Geriatrician in different European countries. European Geriatric Medicine. 2014;5(5):347-51.

14. Gordon AL, Blundell A, Dhesi JK, Forrester-Paton C, Forrester-Paton J, Mitchell HK, et al. UK medical teaching about ageing is improving but there is still work to be done: the Second National Survey of Undergraduate Teaching in Ageing and Geriatric Medicine. Age and Ageing. 2014 March 1, 2014;43(2):293-7.

15. Meiboom AA, de Vries H, Hertogh CMPM, Scheele F. Why medical students do not choose a career in geriatrics: a systematic review. BMC Medical Education. [journal article]. 2015 June 05;15(1):101.

16. Fisher J, Garside M, Brock P, Gibson V, Hunt K, Briggs S, et al. Why geriatric medicine? A survey of UK specialist trainees in geriatric medicine. Age and Ageing. 2017;1(7).

17. Fuller G, Simpson IA. "Modernising Medical Careers" to "Shape of Training" - how soon we forget. BMJ. 2014;348(g2865).

18. Knight PV, Oliver D, Wyrko Z, Gordon AL, Turner G. Frailty related aspects of care are underrecognised in UK medical curriculums. BMJ. 2014;348:g3325.

19. Smith GR, Stein JM, Jones MC. Acute medicine in the United Kingdom: First-hand perspectives on a parallel evolution of inpatient medical care. Journal of Hospital Medicine. 2012;7(3):254-7.

20. Oliver D, Burns E. Geriatric medicine and geriatricians in the UK. How they relate to acute and general internal medicine and what the future might hold? Future Hospital Journal. 2016;3(1):49-54. 
21. Conroy SP, Ansari K, Williams M, Laithwaite E, Teasdale B, Dawson J, et al. A controlled evaluation of comprehensive geriatric assessment in the emergency department: the 'Emergency Frailty Unit'. Age and Ageing. 2013;43(1):109-14.

22. Oo M, Tencheva A, Khalid N, Chan Y, Ho S. Assessing frailty in the acute medical admission of elderly patients. Journal of the Royal College of Physicians Edinburgh. 2013;43:301-8

23. Silvester KM, Mohammed MA, Harriman P, Girolami A, Downes TW. Timely care for frail older people referred to hospital improves efficiency and reduces mortality without the need for extra resources. Age and Ageing. 2013;43(4):472-7.

24. Deschodt M, Claes V, Van Grootven B, Van den Heede K, Flamaing J, Boland B, et al. Structure and processes of interdisciplinary geriatric consultation teams in acute care hospitals: A scoping review. International Journal of Nursing Studies. 2016;55:98-114.

25. Saltvedt I, Mo ES, Fayers P, Kaasa S, Sletvold O. Reduced mortality in treating acutely sick, frail older patients in a geriatric evaluation and management unit. A prospective randomized trial. J Am Geriatr Soc. 2002;50(5):792-8.

26. Ellis $G$, Whitehead MA, O'Neill D, Langhorne $P$, Robinson D. Comprehensive geriatric assessment for older adults admitted to hospital. Cochrane Database of Systematic Reviews 2011(7):Art. No.: CD006211.

27. Barnes DE, Palmer RM, Kresevic DM, Fortinsky RH, Kowal J, Chren M-M, et al. Acute Care For Elders Units Produced Shorter Hospital Stays At Lower Cost While Maintaining Patients' Functional Status. Health Affairs. 2012 June 1, 2012;31(6):1227-36.

28. Chong MS, Chan M, Tay L, Ding YY. Outcomes of an innovative model of acute delirium care: the Geriatric Monitoring Unit (GMU). Clinical Interventions in Aging. 2014;9:603-12.

29. Dewing J, Dijk S. What is the current state of care for older people with dementia in general hospitals? A literature review. Dementia. 2014;15(1):106-24.

30. Bachmann S, Finger C, Huss A, Egger M, Stuck AE, Clough-Gorr KM. Inpatient rehabilitation specifically designed for geriatric patients: systematic review and meta-analysis of randomised controlled trials. BMJ. 2010;340:c1718.

31. Forster A, Young J, Lambley R, Langhorne P. Medical day hospital care for the elderly versus alternative forms of care. Cochrane Database of Systematic Reviews. 2008(4).

32. Palvanen M, Kannus P, Piirtola M, Niemi S, Parkkari J, Järvinen M. Effectiveness of the Chaos Falls Clinic in preventing falls and injuries of home-dwelling older adults: A randomised controlled trial. Injury. 2014;45(1):265-71.

33. Kammerlander C, Roth T, Friedman SM, Suhm N, Luger TJ, Kammerlander-Knauer U, et al. Orthogeriatric service-a literature review comparing different models. Osteoporos Int. 2010;21(4):637-46.

34. Nordström P, Michaëlsson K, Hommel A, Norrman PO, Thorngren K-G, Nordström A. Geriatric Rehabilitation and Discharge Location After Hip Fracture in Relation to the Risks of Death and Readmission. Journal of the American Medical Directors Association. 2016;17(1):91.e1-.e7.

35. Partridge JSL, Collingridge G, Gordon AL, Martin FC, Harari D, Dhesi JK. Where are we in perioperative medicine for older surgical patients? A UK survey of geriatric medicine delivered services in surgery. Age and Ageing. 2014;43(5):721-4.

36. Partridge JSL, Harari D, Martin FC, Dhesi JK. The impact of pre-operative comprehensive geriatric assessment on postoperative outcomes in older patients undergoing scheduled surgery: a systematic review. Anaesthesia. 2014;69:8-16.

37. Schoenenberger AW, Werner N, Bramlage P, Martinez-Selles M, Maggi S, Bauernschmitt R, et al. Comprehensive geriatric assessment in patients undergoing transcatheter aortic valve implantationrationale and design of the European CGA-TAVI registry. European Geriatric Medicine. 2014;5(1):8-13.

38. Pallis AG, Fortpied C, Wedding U, Van Nes MC, Penninckx B, Ring A, et al. EORTC elderly task force position paper: Approach to the older cancer patient. European Journal of Cancer. 2010;46(9):1502-13. 
39. Kenny RA, O'Shea D, Parry SW. The Newcastle protocols for head-up tilt table testing in the diagnosis of vasovagal syncope, carotid sinus hypersensitivity, and related disorders. Heart. 2000;83(5):564-9.

40. Kale R, Menken M. Who should look after people with Parkinson's disease? BMJ. 2004;328(7431):62-3.

41. McLellan A, Gallacher S, Fraser M, McQuillian C. The fracture liaison service: success of a program for the evaluation and management of patients with osteoporotic fracture. Osteoporos Int. 2003;14(12):1028-34.

42. Bloomer A. Continence care in the elderly: whose role is it anyway? GM Journal. 2012;41:633-5.

43. Wyrko Z. Specialties: Geriatric Medicine. London: Royal College of Physicians; 2013; Available from: https://www.rcplondon.ac.uk/sites/default/files/geriatric medicine.pdf.

44. Beswick AD, Rees K, Dieppe P, Ayis S, Gooberman-Hill R, Horwood J, et al. Complex interventions to improve physical function and maintain independent living in elderly people: a systematic review and meta-analysis. The Lancet. 2008;371(9614):725-35.

45. Conroy SP, Stevens T, Parker SG, Gladman JRF. A systematic review of comprehensive geriatric assessment to improve outcomes for frail older people being rapidly discharged from acute hospital: 'interface geriatrics'. Age and Ageing. 2011 July 1, 2011;40(4):436-43.

46. Kojima G. Prevalence of Frailty in Nursing Homes: A Systematic Review and Meta-Analysis. Journal of the American Medical Directors Association. 2015;16(11):940-5.

47. Robbins I, Gordon A, Dyas J, Logan P, Gladman J. Explaining the barriers to and tensions in delivering effective healthcare in UK care homes: a qualitative study. BMJ Open. 2013;3(7).

48. Lisk R, Yeong K, Nasim A, Baxter M, Mandal B, Nari R, et al. Geriatrician input into nursing homes reduces emergency hospital admissions. Archives of Gerontology and Geriatrics. 2012;55(2):331-7.

49. Frank Hoek J, Ribbe MW, Hertogh CMPM, van der Vleuten CPM. The role of the specialist physician in nursing homes: the Netherlands' experience. International Journal of Geriatric Psychiatry. 2003;18(3):244-9.

50. Singler K, Gordon AL, Robertson G, Roller RE. The development of a geriatric postgraduate education assessment instrument using a modified Delphi procedure. Age and Ageing. 2016;45(5):71822.

51. Roller RE, Petermans J. Education and training in geriatrics in the 21st century - where do we come from - where do we go? European Geriatric Medicine. 2015;6(3):205-7.

52. Parks SM, Harper GM, Fernandez H, Sauvigne K, Leipzig RM. American Geriatrics Society/Association of Directors of Geriatric Academic Programs Curricular Milestones for Graduating Geriatric Fellows. Journal of the American Geriatrics Society. 2014;62(5):930-5.

53. Masud T, Blundell A, Gordon AL, Mulpeter K, Roller R, Singler K, et al. European undergraduate curriculum in geriatric medicine developed using an international modified Delphi technique. Age and Ageing. 2014;43(5):695-702.

54. Rowe JW, Fulmer T, Fried L. PReparing for better health and health care for an aging population. JAMA. 2016;316(16):1643-4.

55. Stuck AE, Moser A, Morf U, Wirz U, Wyser J, Gillmann G, et al. Effect of Health Risk Assessment and Counselling on Health Behaviour and Survival in Older People: A Pragmatic Randomised Trial. PLoS Med. 2015;12(10):e1001889.

56. Conroy S, Nickel CH, Jónsdóttir AB, Fernandez M, Banerjee J, Mooijaart S, et al. The development of a European curriculum in Geriatric Emergency Medicine. European Geriatric Medicine. 2016 $7 / / ; 7(4): 315-21$. 